\title{
Prevalence and management of Gaucher disease
}

This article was published in the following Dove Press journal:

Pediatric Health, Medicine and Therapeutics

20 June 2011

Number of times this article has been viewed

\section{T Andrew Burrow \\ Sonya Barnes Gregory A Grabowski \\ The Division of Human Genetics, Cincinnati Children's Hospital Medical Center, and the Department of Pediatrics, University of Cincinnati College of Medicine, Cincinnati, $\mathrm{OH}$, USA}

\begin{abstract}
Gaucher disease is a phenotypically heterogeneous autosomal recessively inherited lysosomal storage disease, resulting from deficient activity of the enzyme glucocerebrosidase (GCase, acid $\beta$-glucosidase) due to mutations in GBA1. Gaucher disease is the prototype for which disease-specific pharmacological therapy was successfully employed. The objective of this review is to provide a comprehensive review and critical examination of the prevalence, pathophysiology, natural history, and management of Gaucher disease.
\end{abstract}

Keywords: lysosomal storage disease, pathophysiology, treatment, inborn errors of metabolism

\section{Introduction}

Gaucher disease is a rare, autosomal recessively inherited lysosomal storage disease, resulting from defective and insufficient activity of the enzyme glucocerebrosidase (GCase, acid $\beta$-glucosidase) due to mutations in GBA1. Decreased catalytic activity and/or instability of GCase leads to accumulation of its major glycolipid substrate, glucosylceramide (GC; glucocerebroside, ceramide- $\beta$-glucoside $)^{1}$ and minor, yet highly toxic substrate, glucosylsphingosine $(\mathrm{GS}),{ }^{2}$ in the lysosomes of monocyte-lineage cells. These lipid-laden macrophages in visceral organs develop into the characteristic, 'Gaucher cells', 3,4 The primary source of storage material in visceral tissues comes from catabolism of glycosphingolipids in the membranes of senescent red and white blood cells. ${ }^{5}$ Gangliosides from neuronal synthesis are the primary source of the accumulated substrates in the central nervous system and lead to neuronal death. ${ }^{6-8}$ Substrate accumulation in 'extralysosomal compartments' in non-macrophage cells has also been proposed. ${ }^{9}$ The pathological mechanisms by which lysosomal glycolipid accumulation leads to disease have yet to be established; however it is likely that diverse mechanisms contribute to this process.

Three phenotypes of Gaucher disease have been described based upon the presence or absence and severity of neurological involvement (Table 1). Type 1, the non-neuronopathic variant, is essentially a macrophage disorder that primarily affects the visceral organs (liver, spleen, and bone marrow) and occasionally lung. The manifestations are highly variable and onset may occur from childhood to adulthood. Types 2 and 3 represent a continuum of central nervous system (CNS) disease and are distinguished by the degree of progression and age at onset. ${ }^{10}$ Gaucher disease type 2 is associated with early ( 3-6 months) onset, severe, rapidly progressive neurological disease and visceral manifestations, and death within the first 1-2 years of age.
Correspondence: Gregory A Grabowsk Cincinnati Children's Hospital Medical Center, Division of Human Genetics, 3333 Burnet Avenue, MLC 4006, Cincinnati, $\mathrm{OH} 45229-3039$, USA

$\mathrm{Tel}+\mathrm{I} 5136367290$

Fax + I $513636226 \mid$

Email greg.grabowski@cchmc.org 
Table I Gaucher disease variants

\begin{tabular}{|c|c|c|c|c|c|c|c|}
\hline \multirow[t]{3}{*}{ Clinical feature } & \multicolumn{7}{|c|}{ Gaucher disease variant } \\
\hline & \multicolumn{2}{|l|}{ I } & \multicolumn{2}{|l|}{2} & \multicolumn{3}{|l|}{3} \\
\hline & Early & Late & Perinatal/lethal & Classical & $3 a$ & $3 b$ & $3 c$ \\
\hline $\begin{array}{l}\text { Age of onset/ } \\
\text { recognition }\end{array}$ & Childhood & Adulthood & Perinatal/Birth & Infancy & Childhood & Childhood & Childhood \\
\hline Hepatomegaly & $+\rightarrow+++$ & $+\rightarrow+++$ & ++ & ++ & $+\rightarrow++$ & +++ & $+\rightarrow++$ \\
\hline Splenomegaly & $+\rightarrow+++$ & $+\rightarrow+++$ & ++ & ++ & $+\rightarrow++$ & +++ & $+\rightarrow++$ \\
\hline Anemia & $+\rightarrow+++$ & $+\rightarrow+++$ & + & $+\rightarrow++$ & + & $+\rightarrow+++$ & + \\
\hline Thrombocytopenia & $+\rightarrow+++$ & $+\rightarrow+++$ & + & $+\rightarrow++$ & $+\rightarrow++$ & $+\rightarrow+++$ & ++ \\
\hline $\begin{array}{l}\text { Cardiac valve/aortic } \\
\text { calcification }\end{array}$ & - & - & - & - & - & - & $+\rightarrow+++$ \\
\hline $\begin{array}{l}\text { Dermatologic } \\
\text { abnormalities }\end{array}$ & - & - & +++ & - & - & - & - \\
\hline Pulmonary disease & $-\rightarrow+++$ & $-\rightarrow+++$ & +++ & +++ & $+\rightarrow++$ & $++\rightarrow+++$ & $-\rightarrow+$ \\
\hline Skeletal disease & $+\rightarrow+++$ & $+\rightarrow+++$ & - & - & + & $++\rightarrow+++$ & + \\
\hline $\begin{array}{l}\text { Neurological } \\
\text { disease }\end{array}$ & - & -* $^{*}$ & +++ & +++ & $++\rightarrow+++^{* *}$ & $+\rightarrow++$ & + \\
\hline Survival & Variable & & $<\mathrm{I}$ month & $<2$ years & $\begin{array}{l}\text { 2nd-4th } \\
\text { decade }\end{array}$ & $\begin{array}{l}\text { Ist-4th } \\
\text { decade }\end{array}$ & $\begin{array}{l}\text { Ist-4th } \\
\text { decade }\end{array}$ \\
\hline
\end{tabular}

Source: Adapted from Grabowski et al."

Notes: The symbols,,++++++ , or - refer to progressively severe involvement from absent $(-)$ to $(\rightarrow)$ very severe $(+++)$ of different clinical manifestations. $*$ Potentially Parkinson disease; ${ }^{* *}$ Intractable myoclonic seizures.

Gaucher disease type 3 is associated with later onset, highly variable neurological disease, and variable degrees of visceral organ involvement. ${ }^{11}$

Although individuals with Gaucher disease are clinically classified into three types, there is a continuum of visceral and/or neurological phenotypes within each type that present major diagnostic challenges, which directly influence the timing and type of treatment modalities. Due to the ever-expanding spectrum of neurological involvement in Gaucher, researchers and clinicians describe patients with both acute and chronic neurological disease as neuronopathic, rather than restricting classification to types 2 or 3 . Although different from types 2 and 3 , those with type 1 can also present with Parkinson's disease symptoms and peripheral neuropathies. ${ }^{12}$ This disease continuum has significant import to the choice and/or institution of treatment in children that present with severe visceral disease, but no apparent CNS involvement. Such patients may be initially diagnosed as type 1, except in combination with the presence of the N370S (discussed below) allele, whereas neurological involvement can appear later in life.

Gaucher disease has been the prototype for successful specific pharmacological therapy. ${ }^{13}$ Enzyme therapy (ET) is safe and efficacious in the treatment of the visceral manifestations of the disease variants. ${ }^{14-16}$ Indeed, since the FDA approved ET in 1991, it has become the standard of care for the treatment of Gaucher disease. ${ }^{17}$ Newer treatment modalities are available or under investigation, including substrate synthesis inhibition, pharmacological chaperone, and gene therapies. These are discussed in Management approaches.

\section{Prevalence}

Gaucher disease is one of the most common lysosomal storage diseases with a worldwide frequency of $\sim 1 / 111,111$ and $\sim 1 / 855$ in the Ashkenazi Jewish population. ${ }^{1,18-20}$ Type 1 disease is the most prevalent form in the western world (US, Europe, Israel, and other European-derived Caucasian populations), accounting for $\sim 94 \%$ of patients. ${ }^{10}$ Types 2 and 3 are neuronopathic variants representing $<1$ and $5 \%$ of patients, respectively, in Europe, North America, and Israel. Types 2 and 3 have much greater frequencies in "non-western" countries including non-Israeli Middle East, Indian subcontinent, China, Japan, and Korea. ${ }^{10,11,21}$ The actual frequencies and variations in the phenotype need to be fully delineated. Importantly, the alleles containing L444P have the greatest worldwide prevalence and are associated with the neuronopathic variants. Despite the higher heterozygote frequency of Gaucher disease type $1(1: 17)$ in the Ashkenazi Jewish population, ${ }^{22}$ type 2 disease is rarely encountered with only 11 published cases to date. ${ }^{23}$

The phenotypic heterogeneity of Gaucher disease has been attributed to many mutations in GBA1. PCR-based screening of 2121 unaffected Ashkenazi Jewish subjects revealed 
4 common mutation genotypes, c.84-85 insG, c1226A $>$ G $(\mathrm{N} 370 \mathrm{~S}), \mathrm{IVS} 2+\mathrm{G} \rightarrow \mathrm{A}, \mathrm{c} .1448 \mathrm{~T}>\mathrm{C}$ (L444P), and collectively account for greater than $96 \%$ of mutations in Ashkenazi Jewish patients, while accounting for less than $75 \%$ of the mutant alleles identified in other "western-world" populations. ${ }^{11,18,19}$ Complete gene sequencing has facilitated identification of new GBA1 mutations in more general populations. ${ }^{24}$ Each of the 320 disease-causing mutations in GBA1 has a frequency that varies between different populations. ${ }^{11,24}$ The majority are missense mutations that result in decreased catalytic activity and/or stability, while nonsense mutations including frame shifts, splice-sites, deletions, alleles resulting from recombination with the pseudogene, gene conversions, and total deletions lead to null alleles.

In the Ashkenazi Jewish population, the two most common alleles are $\mathrm{N} 370 \mathrm{~S}$ and $84 \mathrm{GG}$ with gene frequencies of $1.03 / 1000$ and $0.14 / 1000$, respectively. ${ }^{11,22} 84 \mathrm{GG}$ results in a frame shift, ie, null, and homozygosity has embryonic lethality. The actual prevalence in affected patients is likely to be higher since $\sim 50 \%$ of N370S homozygotes are minimally to asymptomatic and do not come to medical attention. ${ }^{18,25}$ Although N370S and 84GG have high frequency in the Ashkenazi Jewish population, haplotype and linkage disequilibrium studies indicate that the N370S mutation existed in the general European population prior to the founding of the Ashkenazi Jewish population in Europe. In comparison, the $84 \mathrm{GG}$ mutation is in the background of a unique conserved haplotype, indicating a founder effect in the Ashkenazi Jewish population. ${ }^{26}$ Such studies provide a perspective on the existence of the N370S allele in non-Jewish patients at frequencies of 1:21,500 in Portugal, ${ }^{27} 1: 40,247$ in Italy, ${ }^{28}$ $1: 86,000$ in the Netherlands, ${ }^{29}$ and 1.13:100,000 in the Czech Republic. ${ }^{20}$ In non-Ashkenazi Jewish patients, L444P is the most frequent, ${ }^{11}$ which was originally found in neuronopathic Gaucher disease patients. ${ }^{30}$ Interestingly, in the Japanese population, the most prevalent allele, L444P, in either heteroallelic or homoallelic forms, exhibits both neurological and non-neurological phenotypes illustrating the complexity of genotype-phenotype correlation in this population. ${ }^{31}$

Another genotype, D409H/D409H results in a unique neuronopathic variant with calcification or fibrosis of the cardiac valve, corneal opacities, hydrocephalus, and dysmorphic features. ${ }^{32}$ Although originally described in Japan, this phenotype is panethnic, but has a higher frequency in the Middle Eastern neuronopathic variants. As genetic screening for Gaucher disease variants becomes more widespread in the large Asian and Indian populations, additional common and rare variants would be expected, as would additional concentrations of specific mutations in genetic isolates. However, the L444P alleles will likely continue to be the most common mutation(s) worldwide.

The diversity of mutations and the lack of standardized general method for phenotyping have made genotype/phenotype correlations challenging. In the western-world population, predominantly Ashkenazi Jewish, N370S homozygosity does not consistently confer a mild phenotype that manifests in adults since $\sim 50 \%$ of such patients were diagnosed by age 20 years. ${ }^{33}$ However, the incomplete ascertainment of N370S homozygotes complicates these assessments even more. Two conclusions are relatively solid: 1) the presence of a single N370S allele in Gaucher disease patients precludes childhood onset of primary neuronopathic disease ${ }^{11}$ and 2) heteroallelism for N370S and most other nonsense or missense mutations, eg, 84GG or L444P, usually presents in childhood and with significantly progressive disease. ${ }^{25,34}$ Homozygosity for the L444P genotype is significantly associated with development of neuronopathic forms, albeit there are many exceptions. Interestingly, 'true' L444P homozygotes have not been reported in the Ashkenazi Jewish type 2 patients. ${ }^{23}$ Detailed sequence analyses of patients thought to be homozygous for L444P reveal that all had at least one allele resulting from a recombination with the GBA1 pseudogene. ${ }^{35}$ This indicates the importance of using techniques that will accurately detect any allele carrying a L444P mutation when genotyping type 2 patients.

\section{Pathophysiology Enzyme abnormalities}

Mutations in the GBA1, located at chromosome 1q21.3, result in reduced/defective catalytic activity and/or stability of GCase, and lead to diminished GC flux through cells. ${ }^{36}$ Indeed, the crystal structure shows only a single missense mutation involving an active site amino acid; the others are dispersed throughout the protein. ${ }^{37}$

Kinetic and immunologic studies of the residual enzymatic activity from tissues of affected patients with various subtypes and variants of Gaucher disease have indicated several allelic mutations, which result in two general categories of abnormal acid $\beta$-glucosidases: those that have 1) a normal response to enzyme effectors but abnormal stability and/or trafficking and 2) abnormal responses to active site-directed effectors. ${ }^{38,39}$ The N370S enzyme typifies the latter variants. The former enzymes may elicit an unfolded protein response, but the contribution of this to pathogenesis is unclear. Gaucher-like phenotypes also manifest in rare patients deficient in saposin C, an optimizer of GCase activity; ${ }^{40-43}$ three 
had a Gaucher disease type 3-like phenotype and two had no neurological involvement.

\section{Lipid accumulation}

The primary initiator of the pathogenic process is the abnormal accumulation of lysosomal GC and, probably its deacylated analog, glucosylsphingosine. Multiple mechanisms likely potentiate the pathogenic process, but these are not well defined. The substrate, GC, is a ubiquitous, minor, but important lipid component of mammalian tissues cell/organelle membranes. ${ }^{44}$ Importantly, GC is the ultimate glycolipid precursor in the synthesis and degradation of $>300$ neutral glycosphingolipids and gangliosides. GC is synthesized on the cytoplasmic face of the cis-Golgi by UDP-glucose: ceramide glycosyltransferase (EC 2.4.1.80; ceramide glucosyltransferase; glucosycleramide synthase; GlcCer synthase, GCS). ${ }^{45,46}$ The primary source of the accumulating GC in visceral tissues of Gaucher disease patients is the stepwise macrophage lysosomal degradation of higher order glycosphingolipids. This normal process for the turnover of the membranes of senescent red and white blood cells is disrupted by defective GCase. ${ }^{5}$ In comparison, gangliosides from neuronal membranes are the primary source of excess GC in the central nervous system. ${ }^{6-8}$ A minor GCase substrate, GS, can be generated either through GC deacylation or from glycosylation of sphingosine by an unknown enzyme. ${ }^{47,48}$ Although present in very low concentrations in Gaucher disease patients, this lipid is a very potent neurotoxin that alters cellular metabolism ${ }^{49,50}$ and can impair intracellular $\mathrm{Ca}^{2+}$ homeostasis. ${ }^{51,52}$ The exact participation of either GC or GS in these pathogenic processes and their precise delineation are currently unknown, but when understood could provide additional therapeutic targets.

\section{Inflammation}

GC storage in hepatic, splenic, bone marrow, and other tissue macrophages leads to macrophage activation, release of multiple cytokines, chemokines, and the chitinase, chitotriosidase. These pro- and anti-inflammatory agents not only participate in the propagation of the disease process, but can also serve as biomarkers for this disease severity and response to therapy. ${ }^{53}$ Understanding how the evoking of chronic macrophage/inflammatory reactions is initiated and becomes highly disrupted during disease progression is critical to understanding and defining the stage and reversibility of the various manifestations. Recent whole genome transcriptome analyses in mouse models demonstrate large-scale disruption of these finely controlled protective mechanisms.
In humans with Gaucher disease chronic stimulation of the immune system leads to elevations of tumor necrosis factor- $\alpha$ (TNF- $\alpha)$, macrophage colony-stimulating factor (M-CSF), macrophage inflammatory proteins $1 \alpha$ and $1 \beta$ (MIP-1 $\alpha$ and $1 \beta$ ), interleukin $1 \beta$ (IL-1 $\beta$ ), interleukin-6 (IL-6), and interleukin-1 receptor antagonist (IL-1Ra). ${ }^{54-56}$ These cytokines are involved in numerous physiological processes within the body, including hematopoiesis and bone remodeling, which are important in Gaucher disease. These are typified by the cachexia and inanition of many untreated type 3 patients. Indeed, chronic B-cell activation is likely responsible for the 6- to 20-fold increased risk of Gaucher disease type 1 patients developing multiple myeloma. ${ }^{57}$

\section{Clinical history/prognosis}

The phenotypes are a continuum of degrees of involvement, but can be divided categorically into non-neuronopathic (type 1) and neuronopathic (types 2 and 3) variants. Within these categories, great phenotypic heterogeneity exists, and more severe and attenuated variants occur within each type. Within the same family, there can be significant phenotypic variability. Furthermore, with the introduction of effective therapies, phenotypic modification has become evident, such that features observed prior to the development of these therapies are becoming more unusual and new unexpected features are being observed. ${ }^{58}$ The phenotypic spectrum and clinical history of non-neuronopathic and neuronopathic Gaucher disease are briefly presented below and comprehensive reviews are available particularly for more rare complications. ${ }^{11,25}$ The term "natural history" for Gaucher disease is a misnomer since most symptomatic patients have had some type of supportive medical intervention that could alter the disease course. With the advent of specific enzyme therapy, only the treatment course can be documented. The term "clinical history" will be used here to reflect the status of affected individuals who have not received specific enzyme or other therapies.

\section{Non-neuronopathic - type I}

Older literature referred to the type 1 variant as the "adult form" of Gaucher disease, although it is not simply an adult disease. Greater than $50 \%$ of symptomatic, affected individuals are children and adolescents under the age of 20 years. ${ }^{11}$ As large numbers of patients have been analyzed, a continuum of disease severity and progression has become readily apparent. At least two clinical variants are appreciated, 1) early onset (signs and symptoms before 20 years of age), and 2) late onset (signs and symptoms after 20 years 
of age). This clinical categorization has permitted genotype/ phenotype correlations based on the degree of organ involvement and progression and age of onset. Patients with the N370S/L444P or N370S/84GG tend to have earlier and more severe hepatosplenomegaly, and more rapid progression than N370S homozygotes. Patients with the N370S/84GG genotype have a median age of onset/diagnosis of $\sim 6$ years, compared with N370S/L444P (13-15 years), and N370S homozygotes with (28-32 years). Importantly, the ages of onset in these various genotypes have very different clustering. For example, the N370S/84GG and N370S/L444P show relatively tight clusters of ages of onset/diagnosis around the median, whereas N370S/N370S does not cluster and shows a parameter range from $<5$ to $>70$ years of age. Indeed, children with this phenotype have a median age of onset/ diagnosis of 10 years. Also, although $\sim 75 \%$ of Gaucher disease type 1 patients first diagnosed after 50 years have the N370S/N370S genotype, the majority of individuals with Gaucher disease are diagnosed by the second decade. ${ }^{25}$ Critical analyses of such data expose several significant flaws: 1) Genotyping techniques vary and complete analyses of the GBA1 locus was not conducted in all of the patients in the above analyses. For the N370S allele this is critical since a relatively frequent $G B A 1$ mutation, $\triangle 55$, spans a region that is essential for the detection of N370S and if gene primers are not adjusted to exclude this potential mutation, a patient could appear to be homozygous for N370S, but is actually $\mathrm{N} 370 \mathrm{~S} / \Delta 55$. The $\Delta 55$ is a null allele, ie, similar to $84 \mathrm{GG}$. 2) Some of patients included in the database likely were not ascertained by symptomatology, but by family studies and are included even though they are not significantly symptomatic. 3) Finally, the total number of patients with N370S/N370S has been calculated based on assumptions that the other N370S/“other mutation" would be severe enough to have complete ascertainment. While the latter has significant support, a true number for the N370S/N370S would only come from a large-scale population study with complete genotyping. This would probably only be feasible in the Ashkenazi Jewish population and could suffer from a significant ethnic bias. Indeed, a study of Brazilian patients showed a significantly different pattern of disease involvement compared to similar genotypes from the Ashkenazi Jewish or non-Ashkenazi Jewish populations. ${ }^{59}$

The documentation of clinical history of the later onset Gaucher disease type 1 is particularly controversial. Some have suggested that the disease remains relatively stable with little progression over extended periods of time. ${ }^{60}$ However, recently, large longitudinal studies show these patients may have significant progression of visceral and skeletal manifestations, as well as increased risk of malignancies. ${ }^{61}$ Nonetheless, in adults who experience rapid progression of a previously indolent disease, other etiologies, such as malignancy, should be considered before attributing the changes to Gaucher disease. ${ }^{11}$

The occurrence of various clinical abnormalities is important to consider in disease characterization. Again, the majority of data come from western populations with very significant bias toward the Ashkenazi Jewish population because of its high frequency. In the few studies that have been conducted in Gaucher disease type 1 non-Jewish adult populations, there is general, but not specific agreement with results from the Jewish patients. Anemia and thrombocytopenia are present in most patients, except for those with very mild disease and can be quite severe, resulting in associated symptoms (ie, fatigue). Transfusions are rarely required. Thrombocytopenia can result in an increased risk of bruising and bleeding in patients although these can be independent parameters. Clinical bleeding is unusual. Initially, thrombocytopenia is caused by splenic sequestration, but later, 1) splenectomized patients can experience replacement of marrow with Gaucher cells and/or myelofibrosis or 2) nonsplenectomized patients with large spleens can have platelet consumption in infarcted or cystic regions of the spleen. ${ }^{11}$

Hepatomegaly is identified in $>50 \%$ of patients and is particularly prevalent in children. Occasionally, the liver may be massive, filling much of the abdomen, particularly in splenectomized patients. Liver function studies may be slightly elevated. However, overt liver dysfunction is uncommon, except in the presence of co-morbid conditions, such as hepatitis $\mathrm{C}$ or primary hepatocarcinoma. ${ }^{11}$ Therefore, when liver dysfunction occurs, other co-morbidities should be sought.

Asymptomatic splenomegaly is the most frequent presenting sign in Gaucher disease type 1 variants. Occasionally, spleens may be $>50$ times normal volume, resulting in early satiety and mechanical interference with respiratory function. ${ }^{11}$ Although splenectomy results in rapid improvement of these signs/symptoms, anecdotal data suggests that this may cause worsening of bone disease and development of pulmonary hypertension. Additionally, splenic infarction occasionally occurs, which may mimic an acute, surgical abdomen $^{11}$ and should be considered/evaluated in patients presenting as such.

Skeletal disease by radiographic imaging shows that most patients $(75 \%-90 \%)$ have bony lesions, the most common being the Erlenmeyer flask deformity of the distal 
femur. ${ }^{11}$ Other evidence of skeletal involvement may include destruction of the marrow cavity, lytic changes in the cortex, and osteopenia/osteoporosis. Osteopenia is a progressive process that begins in childhood, such that by adulthood significant skeletal disease may exist, particularly in postmenopausal females, although this is not well documented. A severe painful bone infarction or bone crisis occurs in $\sim 10 \%$ of individuals with Gaucher disease, particularly during childhood and adolescence. These crises most frequently involve the femoral head and shaft and over the course of several days evolve from a dull, achy pain to severe pain that is difficult to control with analgesics. Fever and localized swelling and erythema may accompany the pain. Within a few days, the pain diminishes to a dull ache, which may persist for several weeks. ${ }^{11}$ Unfortunately, despite being a significant source of pathology in many patients with Gaucher disease, quantification of skeletal involvement is challenging because of the lack of a single study capable of globally evaluating for skeletal pathology. In addition, there continues to be confusion in the literature between the two compartments of bone that are involved in Gaucher disease: cortical bone and bone marrow. Several sophisticated imaging techniques for quantification of bone marrow changes have fallen short of generalized adoption due to challenges in standardization and availability inside and outside of developed countries. Standard radiographs are useful for quantifying pre-existing lesions, but are very insensitive to short-term changes. DEXA assesses cortical bone mineral density, but the clinical utility and the difficulties in standardization of measurements in children limit its application for static or therapeutic assessments.

Gaucher disease type 1 has been clinically defined by the absence of primary CNS neurological manifestations. The epidemiological data linking Parkinsonism and the GBA1 locus have cast doubt on this definition. A report of increased frequency (36\%) of common neurological findings, eg, dementia, parkinsonism, radiculopathy, and peripheral neuropathy from one center in Israel created a focus for anecdotal and other reports and controversy. ${ }^{62}$ This led to several genetic epidemiological studies directed to determining associations of GBA1 mutations and Parkinson's disease. A large international, multicenter study of 5,691 patients with Parkinson's disease and 4,898 controls revealed an odds ratio of 5.4, across all centers, for persons who had Parkinsonism to also be heterozygous for a $G B A 1$ mutation. ${ }^{63}$ However, this study was limited by variations in mutation analysis techniques among the sites resulting in potential incomplete ascertainment of GBA1 mutations. Nichols and colleagues reported an increased odds ratio (2.6) for Parkinson's disease patients being also heterozygous for a $G B A 1$ mutation. In these studies, Parkinsonism patients with $G B A 1$ mutations tended to have an earlier age of onset of manifestations than those without GBA1 mutations. ${ }^{64}$ From such studies, Parkinsonism patients with null GBAl mutations tended to have earlier onset and more progressive Parkinsonism than those with missense mutations at $G B A .{ }^{65}$

Conversely, studies have indicated an increased risk for development Parkinsonism in individuals with Gaucher disease. ${ }^{66,67}$ In a cohort of 444 patients with Gaucher disease type 1, Bultron and colleagues identified 11 patients with Parkinson's disease. The researchers estimated the adjusted life-time risk ratio of Parkinson's disease in Gaucher disease type 1 to be $\sim 21$ times higher than in the general population, with an increased relative risk in males (up to 31.3-fold) compared to females (up to 13.8-fold). A trend toward earlier onset of Parkinson's disease symptoms among patients with more severe genotypes was identified. Interestingly a significant proportion of individuals with Parkinson's disease were at least heterozygotes for an N370S mutation, which is classically considered to be a neuroprotective mutation. ${ }^{66}$ However, this study had several limitations. It was an observational study in which a group of patients with Gaucher disease were compared to a reference population, with an assumed carrier frequency of 1/100. Additionally, the study population was primarily Ashkenazi Jewish, whereas the reference population was of mixed ethnicity.

These studies are compelling enough to have stimulated intensive research to defining mechanisms of the Parkinsonism and GBA1 mutations. The pathophysiology responsible for the association of Parkinson's disease and Gaucher disease is completely undefined and the reader is referred to Grabowski et $\mathrm{ll}^{11}$ for additional discussion.

An increased risk of cancer, particularly involving the blood-forming organs, ie, multiple myeloma and hematologic cancers (leukemia, Hodgkin's, and non-Hodgkin's lymphoma) has been well established in patients with Gaucher disease. Indeed, the lifetime risk of multiple myeloma has been estimated to be 25 times that of the general population. ${ }^{61}$ However, other forms of cancer, including bone tumors (ie, osteoblastoma) and solid organ cancers may also be more common in individuals with Gaucher disease, compared to the general population. ${ }^{11,61}$ Indeed, the overall cancer risk was estimated at 1.8 times that of the general population. ${ }^{61}$ The etiology for this manifestation in Gaucher disease is unclear. However, given the relatively high frequency of cancer in the general population, occurrence of certain type (ie, solid organ cancers) within the Gaucher disease 
population may be incidental and associated with other factors (ie, environmental) rather than Gaucher disease.

Using data from the ICGG Gaucher registry, life expectancy for patients with Gaucher disease type 1 was estimated to be $\sim 8.9$ years (68.2 years vs 77.1 years) shorter than of those without Gaucher disease, regardless of gender. Interestingly, splenectomized patients had a $\sim 13.2$ year decrease in calculated life expectance from birth compared to the reference population, whereas in non-splenectomized patients, the estimated life expectancy was decreased by 5.1 years. ${ }^{68}$ This skewing may be related to surgical complications from splenectomy, but also to the recognized risk of future complications in splenectomized patients (see above). The increased risk of certain forms of cancer may contribute to the late morbidity and mortality within this population. ${ }^{69}$ However, this was a review of registry data, which relies on voluntary reporting from participating centers. Therefore, it is possible that some of the deceased patients were misclassified and actually had Gaucher disease type 3, in which case younger age of death is not terribly surprising. Additionally, the registry primarily enrolls patients with manifestations severe enough to come to medical attention, providing a rather myopic view of the phenotypic variability/degree of severity of the disease.

The overall connections between the fundamental molecular events that occur in Gaucher disease type 1 over decades and the complications of more common diseases clearly remains to be raveled. Challenging large-scale prospective studies are needed to gain sufficient epidemiological data and insights. Such studies are likely impossible in the era of direct treatment of Gaucher disease as many of the manifestations are partially or completely eliminated.

\section{Neuronopathic variants}

The neuronopathic Gaucher disease variants are also characterized by a continuum of phenotypes, with significantly variable ages of onset, features, and rates of progression. For practical and prognostic purposes, two variants, types 2 (acute) and 3 (sub acute), are classically distinguished based upon the severity and rapidity of progression of neurological involvement, with the former being more rapidly progressive with a mean age of death of $<1$ year.

As awareness of Gaucher disease expands, worldwide evidence is supporting the contention that neuronopathic disease variants represent the majority of patients. An increasingly broad spectrum of mutations is being identified in these variants. The Swedish Norbottinian type 3 variant is instructive (also see page 8). These patients all descended from a single founder with the L444P GBA1 allele. Within this relatively ethnically homogenous the variance of the phenotype is from a very early onset disease with rapid neurodegeneration to slowly progressive eye movement abnormalities and survival for 4 to 5 decades. This degree of variation and the plethora of mutations have made genotype/phenotype correlations particularly difficult to develop. In addition, the differences in the methods for genotyping, ie, mutation specific vs whole gene sequencing create more complications. The L444P mutation is commonly identified by direct detection of the single base substitution. However, many L444P containing alleles also have additional mutations that arise from recombination with the pseudogene, ${ }^{11}$ thus, the conclusion that an individual with type 2 or 3 disease may appear to be homozygous for L444P, but in actuality may be either L444P/ L444P or L444P/L444P plus additional point mutations. The latter is nearly confined to the type 2 population, whereas the former can occur in types 2 or 3. The L444P plus other additional point mutations, the so-called rec or recombinant alleles are essentially null. The presence of two recombinant alleles appears to predispose to hydrops fetalis and prenatal lethality. Finally, homozygosities for the c.533delC, S196P, R131 L, G202R, or H311R mutations are associated with Gaucher disease type $2{ }^{35}$

\section{Gaucher disease type 2}

Gaucher disease type 2 is the most severe form of Gaucher disease and represents a continuum from prenatal/neonatal onset, the perinatal-lethal variant, to development of manifestations with the first year of life, often by 3-6 months of age (classic type 2 variant).

Prenatal evidence of fetal disease may include poor fetal movement, intrauterine growth retardation, cardiomegaly, non-immune hydrops fetalis (with and without ascites), hepatomegaly and splenomegaly, and evidence of joint contractures/arthrogryposis (as a result of fetal akinesia). ${ }^{11,70}$ Hydrops fetalis is associated with an increased risk of fetal death and premature delivery. In those who survive to delivery, death frequently occurs within the first week of life. Conversely, when hydrops is absent, the fetus is more capable of reaching a full term delivery, with rapidly worsening neurological function leading to death within three months. ${ }^{11,70}$

At birth, features such as those described above may again be identified. Hematological abnormalities, specifically, thrombocytopenia, and less frequently anemia, as well as skin abnormalities, ie, icthyosis, ( $40 \%$ of cases) may be observed. Failure to thrive eventually progresses into cachexia. Dysmorphic features may be observed, 
including low set ears, small nose with a flat nasal bridge and anteverted nares, and hypertelorism. Less frequently, microstomia, everted lips, and micro-retrognathia may also be observed. ${ }^{11,70}$

Severe neurological (cortical and brainstem) dysfunction becomes apparent at or shortly after birth with findings including arthrogryposis, microcephaly, and neonatal distress. The infants exhibit swallowing dysfunction, abnormal posturing, and central apnea. ${ }^{70}$ Death occurs within the first month of age due to severe neurological involvement.

In the classic variant, babies usually appear normal at birth. However, the disease manifests by 3 to 6 months of age with progressive visceral and neurological disease. Splenomegaly is the most frequent finding (59\% of cases) to be detected at the onset of disease. It is followed by other visceral disease manifestations as hepatomegaly, failure to thrive leading to cachexia, pulmonary disease (due to chronic aspiration and Gaucher cell infiltration), and thrombocytopenia with or without anemia. ${ }^{71}$ Unlike non-neuronopathic Gaucher disease, skeletal disease is usually not as apparent as other visceral manifestations.

Neurological involvement is progressive and characterized by brainstem and cortical dysfunction. The most frequent initial bulbar signs include hyperextension of the neck, swallowing impairment, and strabismus. The former ultimately progresses to opisthotonus, which worsens with stimulation. Motor dysfunction is characterized by hypertonia and hyperreflexia. Other neurological signs may include epilepsy, trismus, stridor, and progressive microcephaly. Cognitive involvement is universal and usually begins as developmental stagnation, but later progresses to neurological degeneration. Untreated affected individuals do not develop the ability to speak, stand, or walk. Death usually occurs by two years of age, due to central apnea or pulmonary disease. ${ }^{11,71}$

\section{Gaucher disease type 3}

Gaucher disease type 3 is more heterogeneous than Gaucher disease type 2 and usually follows a more slowly progressive course than the latter. Indeed, evidence of neurological involvement may not occur for years to decades, as indicated above for the Swedish variant. This can result in an incorrect initial categorization of the phenotype as type $1 .{ }^{11}$ Because of the significant variability in degree of visceral and neurological disease manifestations among patients with Gaucher disease type 3, determinations of phenotype based upon genotype are quite challenging.
Because of this, the prognostic information is very limited and survival with significant quality of life can be for several decades.

This variant is often separated into three subtypes: $3 a, b$, and c. Those with type 3 a demonstrate progressive myoclonic epilepsy and dementia. Those with aggressive systemic disease and supranuclear gaze palsy, with or without other neurological manifestations are classified as having type $3 \mathrm{~b}$. Finally, individuals with type $3 \mathrm{c}$ demonstrate unique features, including impaired horizontal ocular saccades, corneal opacities, and cardiac valvular calcification. This phenotype is specific to individuals who are homozygous for the D409H allele. $^{11}$

In one study of 22 patients with Norrbottnian type 3 , the diagnosis was made between 8 months and 14.5 years (median age 25 months). The median age of first manifestation was $\sim 12$ months, at which time patients typically demonstrated normal intelligence, short stature, and splenomegaly. However, with time neurological abnormalities (see below) developed in many of these patients. ${ }^{72}$

With regards to neurological involvement, horizontal supranuclear gaze palsy is an early universal finding. In such patients, the myoclonic jerks become stimulus sensitive and increase with voluntary movement. Over time, the myoclonic seizures become more severe and eventually become refractory to anticonvulsive therapy. Affected individuals also may experience ataxia, spasticity, and $\sim 60 \%$ have cognitive dysfunction. ${ }^{11,73}$

Lifespan in individuals with this phenotype is shortened. Indeed, the average age of death in individuals in the Swedish type 3 variant was 9.8 years, and was the result of progressive deterioration of their disease, ${ }^{72}$ but survival beyond the third decade occurs commonly.

In addition to the above described type 3 variants, an intermediate phenotype with onset later than typically observed in individuals with type 2 , but with more severe neurological involvement than usually observed with type 3 disease has been described. ${ }^{74}$ Nine such patients diagnosed between 8 and 20 months of age had severe neurological disease by 12.6 months, including horizontal supranuclear gaze palsy and refractory myoclonic seizures. These seizures heralded a rapid decline in neurological function, with associated ataxia, dementia, and opisthotonus, and death on average by 3.3 years, because of progressive brainstem involvement and aspiration pneumonia. The lack of clear predictability from genotyping limits the generality of statements related to survival and degrees of involvement and mental impairment. Until environmental and/or other gene modifier effects 
are discovered, progression will be dependent on individual evaluations for progression.

\section{Diagnosis}

Gaucher disease should be considered in the differential diagnosis in individuals with clinical features suggestive of the disease, including hepatomegaly, splenomegaly, anemia, thrombocytopenia, and skeletal disease. The diagnosis is frequently suspected due to detection of characteristic pathological findings (lipid laden cells of monocyte/macrophage origin, termed Gaucher cells) in a variety of tissues in the course of a diagnostic evaluation (ie, liver or bone marrow biopsies performed to evaluate hepatic or hematologic abnormalities, respectively). However, the diagnosis should not be based solely upon the presence of pathologic findings, as similar cells, pseudo-Gaucher cells, may be observed in a variety of other conditions, including multiple myeloma, lymphoma, and acute lymphocytic leukemia. Instead, the diagnosis is only established by demonstration of deficiency GCase activity and/or by identification of pathogenic GBAl mutations.

The "gold-standard" for diagnosis is finding deficient activity of acid $\beta$-glucosidase activity in nucleated cells, ie, peripheral leukocytes, cultured fibroblasts, or amniocytes; the enzyme is not normally present in serum/plasma. The enzyme activity is assessed using a fluorometric artificial substrate, ie, 4-methylumbilliferyl- $\beta$-glucoside. ${ }^{11}$ Although patients with neuronopathic phenotypes of Gaucher disease may have lower enzyme activities than those with non-neuronopathic phenotypes, predictions of disease severity cannot reliably be based upon the percentage of residual enzyme activity. Significant overlap $(\sim 30 \%-40 \%)$ between the enzyme activities in carriers and non-carriers exists; therefore, enzyme analysis is not recommended for determining an individual's carrier status.

Molecular genetic analysis is the standard for confirmation of the diagnosis of Gaucher disease, and offers certain advantages over enzyme assays, including superior DNA stability, and accurate carrier status determination, and potential to establish certain genotype/phenotype correlations. Additionally, mutation analysis allows for some genotype/ phenotype predictions with the caveats outlined above. Furthermore, once the pathogenic mutations are identified in a family, their detection can be used for diagnosis of the disease and the detection of heterozygotes. The cost of DNA sequencing is now such that individual single nucleotide detection is being supplanted by full gene sequencing, even in families in whom the mutations are known. Indeed, given the relative frequency of certain rare alleles, full gene sequencing is essential to accurate diagnosis. The presence of a rare complete gene deletion make parental studies essential as does the $\Delta 55$ or other such alleles.

\section{Management approaches}

Comprehensive evaluation and development of a personalized management plan is essential for the optimal care of affected patients with all variants of Gaucher disease. These supportive measures and severity assessment are addressed in recent reviews and papers. ${ }^{75-81}$ Here, the variant of specific treatment choices available or soon to be available will be highlighted.

Available and potential specific therapies for Gaucher disease include enzyme therapy (ET), substrate reduction therapy (SRT), and pharmacological chaperone therapy or enzyme enhancement therapy (EET). ET relies on supplying a specific exogenous enzyme to cells. Currently, this is accomplished by intravenous infusions of purified recombinant enzyme for uptake into the target cells involved in a specific disease. For Gaucher disease type 1, the target is the macrophage. In comparison, SRT seeks to decrease the synthesis of the offending substrate so that the decreased catalytic capacity present in the affected person's cells can more adequately decrease the total cellular content of these substrates. EET seeks to stabilize or improve compartmentalization of an abnormal enzyme and thereby increase the catalytic capacity of the mutant enzyme within the lysosome.

\section{Enzyme replacement therapy}

ET for Gaucher disease was FDA approved in 1991 (Ceredase $^{\circledR}$, alglucerase; Genzyme Corporation, Cambridge, MA, USA). Because of the clear limit on the amount of this human placental GCase and potentials for bio-contaminants, recombinant human GCase, imiglucerase $\left(\right.$ Cerezyme $^{\circledR}$, imiglucerase, Genzyme Corporation, Cambridge, MA, USA) was developed and was FDA approved in 1994 and subsequently in other countries. Imiglucerase is produced in bioreactors that culture Chinese hamster ovary $(\mathrm{CHO})$ cells expressing human GCase. Imiglucerase differs from alglucerase by having an arginine to histidine substitution at amino acid residue 495. The oligosaccharide structures are very similar. Over the past $\sim 15$ years, ET with imiglucerase has become the standard of care for treatment of significantly symptomatic Gaucher disease type 1, and safety and efficacy data and dose response characteristics are available on $>5,000$ such patients. ${ }^{82,83}$ 
VPRIV $^{\circledR}$ (velaglucerase alfa, Shire Human Genetic Therapies, Inc, Cambridge, MA, USA) was FDA approved in 2010 for ET in Gaucher disease patients by the FDA and European Medicines Agency. Velaglucerase alfa is produced in a human fibrosarcoma cell line using Gene-Activation ${ }^{\circledR}$ technology, has different N-glycosylation structures than imiglucerase, and has the amino acid sequence of placental human GCase. ${ }^{84}$ Review of published clinical trials for velaglucerase alfa demonstrates that it is safe and efficacious in the treatment of Gaucher disease. Furthermore, studies indicate that velaglucerase alfa and imiglucerase are clinically equivalent with regards to hematologic, hepatic, and splenic manifestations. ${ }^{85,86}$

Uplyso $^{\circledR}$ (Taliglucerase alfa, prGCD, Pfizer Inc, New York, NY, USA) is in clinical trials. Taliglucerase alfa is a human GCase produced in carrot cells and contains the same arginine to histidine substitution at amino acid residue 495 as imiglucerase. In addition, taliglucerase also contains additional $\mathrm{N}$ - and $\mathrm{COOH}$ - terminal non-natural amino acids ${ }^{87}$ and specific plant-derived $\beta$-xylose and $\alpha$-fucose in the oligosaccharide structures. As of this writing, detailed results of clinical trials of taliglucerase alfa have not been released.

Despite differences in cell culture/manufacturing process, glycosylation patterns, and amino acid structure among the preparations, these differences do not appear to significantly affect their clinical properties, although data are limited for taliglucerase alfa. Recent results suggest that the differential glycosylation patterns and amino acid structures affect antibody conversion rates in patients. A smaller (1\%) IgG conversion rate for velaglucerase alfa was reported compared to $\sim 13 \%-15 \%$ in those treated with imiglucerase. ${ }^{88}$ However, the lack of a standardized antibody assay limits the interpretation of these data. In one study, three patients were positive for IgG antibodies to imiglucerase at screening; these antibodies did not react with velaglucerase alfa and no patients developed $\mathrm{IgG}$ antibodies to velaglucerase alfa. ${ }^{88}$ This is a rather surprising finding given that the enzymes are essentially identical except for their glycosylation patterns. Therefore, it is unclear why antibodies for imiglucerase would not also recognize velaglucerase alfa. However, differences in glycosylation patterns must be considered as a potential explanation.

Because taliglucerase alfa is produced in a plant (carrot) cell, and, consequently, harbors a unique glycosylation pattern, there has been concern about potentially increased risk of immunogenicity of this preparation, compared to preparations produced in mammalian cells. The limited data on the clinical safety and efficacy of taliglucerase alfa, do not allow for conclusions about comparability.

Despite the immense success of ET, it has certain disadvantages. ET requires intravenous access for infusions over a few hours every two weeks. Many patients find this to be very intrusive. Availability/maintenance of adequate peripheral or central venous access is also challenging, and the costs for supplies and skilled nursing (in addition to the considerable cost for the drug itself) to maintain the access are significant. Unfortunately, many patients, particularly those in developing countries, do not have access to the specialized care required to provide ET. Recent production and manufacturing issues with imiglucerase have highlighted the challenges of partially treating the disease during medication shortages. Indeed, during this tumultuous period, many experienced objective (ie, decreased platelets, hemoglobin) and subjective (ie, fatigue, bone pain) worsening of their disease. Furthermore, certain tissues, ie, lung, and lymph nodes, have been found to be poorly accessible to the recombinant enzyme, resulting in diminished/absent therapeutic response in these tissues. ${ }^{58}$ Importantly, ET does not have an impact on the neurological manifestations of neuronopathic Gaucher disease since the enzyme does not enter the CNS in therapeutic levels.

\section{Substrate reduction therapy}

Oral therapies have been explored using small molecules. The concept of substrate reduction therapy as a potential therapeutic option for Gaucher disease was first proposed by Norman Radin in $1976 .{ }^{89}$ Since that time, two different preparations, Miglustat ${ }^{\circledR}$ (N-butyldeoxynojirimycin; Zavesca ${ }^{\circledR}$, Actelion Pharmaceuticals, Allschwil, Switzerland) and Eligustat tartrate (Eliglustat ${ }^{\circledR}$, Genzyme Corp, Cambridge, MA, USA) have been developed and are either licensed for use in Gaucher disease type 1 (miglustat) or in clinical trials (eliglustat tartrate).

Miglustat is an N-alkylated imino sugar that functions as a competitive glucosylceramide synthase inhibitor. It is licensed for use in the United States, Europe, and Israel in adults with mild to moderate Gaucher disease type 1 for whom ET is unavailable or contraindicated.

In the initial clinical trial of miglustat, 28 patients were treated with the drug in an open-label, non-comparative, multicenter study. Twenty-two patients completed the 12-month study. Six patients withdrew from the study because of gastrointestinal complaints (2), personal reasons (2), or severe pre-existing disease (2). At the conclusion of the study, mean liver and spleen volumes reduced 
from pretreatment state by $\sim 19 \%(P<0.001)$ and $12 \%$ $(P<0.001)$, respectively. Hematological variables improved slightly. Mean chitotriosidase concentrations decreased by $\sim 16.4 \%$ ( $P<0.001) .{ }^{90}$ Eighteen patients were subsequently enrolled in a 24-month extension study. After 36 months on miglustat, continued improvements in liver and spleen volume, platelets, and hemoglobin were reported..$^{91}$

In a separate study, 36 patients who had received a minimum of 2 years of ET were randomized into one of three treatment groups: continued ET, combination therapy with ET and miglustat, and a switch from ET to miglustat. Thirty-three patients completed the 6-month trial. During this period, no significant differences in liver or spleen volume were noted with the exception of a significant decrease in liver volume in the combination treatment group $(P=0.047)$ compared to the imiglucerase group. Although no significant differences in mean change in hemoglobin level were seen between groups, a significant difference in platelet counts was observed between the miglustat and imiglucerase treatment groups $(P=0.035)$, but not with the combination therapy group. Although mean serum chitotriosidase levels, a biomarker, remained relatively stable between the groups, increases in this biomarker were observed in some patients. Three patients were discontinued from the study during the 6-month randomization phase due to adverse events ( 2 in the miglustat monotherapy group and 1 in the combination therapy group). ${ }^{92}$

Of the 33 patients who completed the 6-month randomization period, 29 enrolled in an 18-month extension phase, during which time they received miglustat monotherapy. Fifteen patients completed the study (6/14 discontinued due to adverse events). Although no significant changes in clinical parameters were observed in those completing the 18-month extension study, some patients experienced decreasing hemoglobin levels, platelet counts, and increased chitotriosidase levels. ${ }^{92}$ This suggests that miglustat monotherapy might not be sufficient to maintain the same adequate disease control in all patients.

The effects of miglustat on bone disease in patients with Gaucher disease type 1 have also been studied. The results of a pooled analysis of three open-label clinical trials suggested that miglustat monotherapy might reduce the incidence of bone pain and improve bone mineral density, including those with a history of splenectomy and/or osteoporosis. ${ }^{93}$ However, only adults (a majority of whom presumably had evidence of skeletal disease) were evaluated in these studies. Therefore, it is unclear whether chronic miglustat treatment, initiated prior to the onset of significant skeletal disease, would stabilize and prevent progression of skeletal disease in these patients.

Miglustat has been shown to cross the blood-brain barrier to a limited degree. ${ }^{94}$ Schiffmann and colleagues evaluated the effects of miglustat, concomitant with ET, in 30 patients with Gaucher disease type 3. Visceral disease remained stable in patients in the combination and ET groups. Miglustat did not have any significant benefits on neurological manifestations. ${ }^{95}$ Interestingly anecdotal reports have been published suggesting that combination therapy of miglustat and imiglucerase may be at least somewhat beneficial in treating the visceral and neurological manifestations in some patients with neuronopathic disease. ${ }^{96}$ Therefore the use of combination therapy in Gaucher disease, particularly neuronopathic variants, requires additional evaluation.

Despite the relative convenience for many patients treated with miglustat, this treatment has drawbacks. Gastrointestinal signs, ie, osmotic diarrhea (presumably due to inhibition of intestinal disaccharidase), weight loss, bloating, and belching, are reported in up to $\sim 80 \%$ of patients and may become severe in some patients. These are reportedly worse within the first few months of therapy, may respond to dietary modification, ${ }^{97}$ and frequently improve within six months to one year of therapy. Regardless, post-authorization monitoring indicates that a significant number $(\sim 30 \%)$ of patients discontinue treatment, predominantly for gastrointestinal signs $(\sim 12 \%)$, and most frequently within the first six months of therapy. ${ }^{98}$ In addition, neurological signs, including tremor ( $\sim 30 \%$ of patents), and peripheral neuropathy are also reported. These issues respond to discontinuation of therapy or dose reduction. Cognitive dysfunction has been reported in some participants during clinical trials, but this was not related to the medication..$^{90,91}$

The safety and efficacy of miglustat in children has not been formally evaluated; therefore, the long-term safety and efficacy of the medication, particularly in preventing the longterm complications of Gaucher disease, are unclear. However, one would expect that children would experience many of the same side effects observed in adults (ie, significant gastrointestinal manifestations), which is certainly undesirable to many families and caregivers of these patients.

Eligustat tartrate is a specific, competitive glucosylceramide synthase inhibitor that has a similarity to the ceramide component of glucosylceramide. Phase I and II clinical trials of eliglustat tartrate have been completed, and phase III clinical trials are currently underway. In the multinational, open-label, single-arm phase II study, the efficacy, safety, and pharmacokinetics of eliglustat tartrate (dosed at 50 or 
$100 \mathrm{mg}$ by mouth two times per day based upon plasma drug concentrations) were evaluated in 26 treatment naïve patients with Gaucher disease type 1 after 52 weeks of therapy. ${ }^{99}$ The composite primary efficacy endpoint included improvement in at least two of three disease manifestations (splenomegaly, thrombocytopenia, and/or anemia) after 52 weeks. Twenty patients (87\%) completed the 1-year study and continued in a study extension for an additional year. At the end of the second 52 weeks of study, $77 \%$ of patients met the composite primary efficacy end point. Indeed, statistically significant improvements in mean hemoglobin $(1.62 \mathrm{~g} / \mathrm{dL} P<0.001)$, platelet count $(40.3 \% P<0.001)$, spleen volume $(-38.5 \%$ $P<0.001)$, and liver volume $(-17 \% P<0.001)$. These results are similar to those observed in patients treated with ET over a comparable time course. Furthermore, no bone crises or clinically significant changes were noted for mobility, bone pain, or skeletal X-ray assessments, and the mean Z-score and T-score for lumbar spine bone mineral density improved significantly ( $\sim 0.31 \mathrm{Z}$-score). Elevated biomarkers decreased by $35 \%-50 \%$ and plasma glucosylceramide and ganglioside GM3 normalized.

Twenty patients completed a total of two years of therapy. In these, $\sim 85 \%$ of patients met $>3$ of 4 therapeutic goals. Statistically significant $(P \leq 0.01)$ percent improvements from baseline were observed for platelet count $(+81 \%)$, hemoglobin level $(+20 \%)$, spleen volume $(-52 \%)$, liver volume $(-24 \%)$, and lumbar spine bone mineral density $(+7.8 \%)$. Greatest improvements in hemoglobin and organ-volume reductions were observed in individuals with more extensive baseline disease manifestations. For those who had lytic lesions at the beginning of the study ( 9 patients), these lesions remained stable and no new lesions were detected. Additionally, a 75\% reduction in chitotriosidase and chemokine CCL18 levels were identified, and plasma glucosylceramide and ganglioside GM3 levels remained normal. ${ }^{100}$

In general, the eliglustat tartrate is well tolerated at therapeutic doses. In some of the patients who received doses of $\geq 10 \mathrm{mg} / \mathrm{kg}$, modest increases in PR, QRS, and QT/QTc intervals were observed at the time of maximum plasma drug concentrations. However, neither therapeutic (200 mg) nor supratherapeutic $(800 \mathrm{mg})$ doses of eliglustat had a significant effect on cardiac conduction. ${ }^{101}$

In the phase II clinical trial, up to $\sim 90 \%$ of study participants experience adverse events. However, most events were mild to moderate in intensity and considered unrelated to treatment. These included urinary tract infection; increased blood pressure; gastrointestinal complaints, ie, abdominal pain and diarrhea; headache; and palpitations. The high frequency of diarrhea observed in patients taking miglustat was not reported during the eliglustat clinical trials. This is not surprising as in vitro studies have demonstrated that eliglustat does not inhibit intestinal disaccharidases at concentrations in the high micromolar range, whereas miglustat does. ${ }^{102}$ Two participants showed evidence of peripheral neuropathy during nerve conduction studies; however, both patients remained asymptomatic and had normal neurologic examinations after 3 years of testing. Six patients were withdrawn from the phase II study: pregnancy $(n=3)$ (two of which resulted in spontaneous abortion); asymptomatic non-sustained ventricular tachycardia $(\mathrm{n}=2)$; and progression of pre-existing osteonecrosis $(n=1)$. However, upon retrospective analysis, it was concluded that the asymptomatic non-sustained ventricular tachycardia was related to the study medication..$^{99,100}$ Two phase III trials are underway.

\section{Conclusions}

Gaucher disease is an exceptionally heterogeneous disorder within and among the neuronopathic and non-neuronopathic phenotypes, and onset is from infancy to adulthood. Although it is generally segregated into three variants based upon the absence or presence and severity of neurological involvement, such a classification system fails to recognize the degree of variability that exists in this disease. Indeed, many consider the disease as a continuum of phenotypic features, from mild to severe.

To date, two forms of ET are approved for use in Gaucher disease (imiglucerase and velaglucerase alfa), while another is currently undergoing clinical trials (taliglucerase). Additionally one form of substrate reduction therapy (miglustat) is currently approved for use in specific circumstances, while another (eliglustat) is undergoing clinical trials. Such interest in development of therapeutics for an orphan disease has been remarkable, but unsurprising, given that the treatment of Gaucher disease represents a one billion US dollar market to the pharmaceutical industry. Indeed, the clinical and financial success of alglucerase and imiglucerase provided the impetus for additional therapeutic developments in other lysosomal diseases and has spurred interest by large pharmaceutical companies in this and other orphan disease spaces.

The need for alternative forms of therapy has been particularly highlighted recently by manufacturing issues that resulted in shortages of imiglucerase and caused significant challenges for the individuals with Gaucher disease and their managing physicians. The availability of alternative therapies provides certain advantages. For example, development of alternative sources of therapy for Gaucher 
disease provides clinicians with a larger armamentarium of therapies from which they may select the most appropriate preparation to provide optimal care for their patients. This allows clinicians to provide personalized medicine to their patients, a concept that is unique in the treatment of genetic diseases. Unfortunately, all of these therapies are quite costly. While many hope that development of alternative therapies will promote competition and lower the overall cost of these therapies, this, unfortunately, has yet to become a reality.

Recent significant advances in the treatment of Gaucher disease makes this a very exciting period for individuals with Gaucher disease and those who treat them. Importantly and unlike other disease states, large comparative studies of efficacy and safety are not possible in this orphan disease. Comparisons rest on conclusions drawn from relatively few uncontrolled studies that have non-standardized assessment parameters, measurements, and outcomes. As such, the continued accrual of treatment data and assessments will hopefully form the bases for informed decisions, rather than being based on pharmaceutical propaganda. Thus, the promise of future advancements that provide hope for continued progress in the treatment of this disease rests on the continuing vigilance of the physicians and their patients in rigorous treatment outcomes and solid data.

\section{Disclosure}

No conflicts of interest were declared in relation to this paper.

\section{References}

1. Aghion E. La maladie de Gaucher dans l'enfance. [PhD Thesis]. Paris, 1934.

2. Miyatake T, Suzuki K. Additional deficiency of psychosine galactosidase in globoid cell leukodystrophy: an implication to enzyme replacement therapy. Birth Defects Orig Artic Ser. 1973;9(2):136-140.

3. Burns GF, Cawley JC, Flemans RJ, et al. Surface marker and other characteristics of Gaucher's cells. J Clin Pathol. 1977;30(10):981-988.

4. Boven LA, van Meurs M, Boot RG, et al. Gaucher cells demonstrate a distinct macrophage phenotype and resemble alternatively activated macrophages. Am J Clin Pathol. 2004;122(3):359-369.

5. Kattlove HE, Williams JC, Gaynor E, Spivack M, Bradley RM, Brady RO. Gaucher cells in chronic myelocytic leukemia: an acquired abnormality. Blood. 1969;33(2):379-390.

6. Brady RO, Barton NW, Grabowski GA. The role of neurogenetics in Gaucher disease. Arch Neurol. 1993;50(11):1212-1224.

7. Gornati R, Berra B, Montorfano G, et al. Glycolipid analysis of different tissues and cerebrospinal fluid in type II Gaucher disease. J Inherit Metab Dis. 2002;25(1):47-55.

8. Nilsson O, Svennerholm L. Accumulation of glucosylceramide and glucosylsphingosine (psychosine) in cerebrum and cerebellum in infantile and juvenile Gaucher disease. J Neurochem. 1982;39(3):709-718.

9. Elleder M. Glucosylceramide transfer from lysosomes-the missing link in molecular pathology of glucosylceramidase deficiency: a hypothesis based on existing data. J Inherit Metab Dis. 2006;29(6):707-715.
10. Charrow J, Andersson HC, Kaplan P, et al. The Gaucher registry: demographics and disease characteristics of 1698 patients with Gaucher disease. Arch Intern Med. 2000;160(18):2835-2843.

11. Grabowski GA, Petsko GA, Kolody EH. Gaucher disease. In: Scriver C, Beudet AL, Sly WS, et al, editors. The Online Metabolic and Molecular Bases of Inherited Disease-OMMBID. Available at: www.ommbid.com. New York, McGraw-Hill, Chap 146. Accessed 27 Mar 2011.

12. Cherin P, Rose C, de Roux-Serratrice C, et al. The neurological manifestations of Gaucher disease type 1: the French Observatoire on Gaucher disease (FROG). J Inherit Metab Dis. 2010;33(4):331-338.

13. Brady RO, Pentchev PG, Gal AE, Hibbert SR, Dekaban AS. Replacement therapy for inherited enzyme deficiency. Use of purified glucocerebrosidase in Gaucher's disease. N Engl J Med. 1974;291(19):989-993.

14. Brady RO, Barton NW. Enzyme replacement therapy for Gaucher disease: critical investigations beyond demonstration of clinical efficacy. Biochem Med Metabol Biol. 1994;52(1):1-9.

15. Grabowski GA, Barton NW, Pastores G, et al. Enzyme therapy in type 1 Gaucher disease: comparative efficacy of mannose-terminated glucocerebrosidase from natural and recombinant sources. Ann Intern Med. 1995;122(1):33-39.

16. Murray GJ, Doebber TW, Shen TY, et al. Targeting of synthetically glycosylated human placental glucocerebrosidase. Biochem Med. 1985;34(2):241-246.

17. Barton NW, Brady RO, Dambrosia JM, et al. Replacement therapy for inherited enzyme deficiency-macrophage-targeted glucocerebrosidase for Gaucher's disease. N Engl J Med. 1991;324(21):1464-1470.

18. Beutler E, Nguyen NJ, Henneberger MW, et al. Gaucher disease: gene frequencies in the Ashkenazi Jewish population. Am J Hum Genet. 1993;52(1):85-88.

19. Zimran A, Gelbart T, Westwood B, Grabowski GA, Beutler E. High frequency of the Gaucher disease mutation at nucleotide 1226 among Ashkenazi Jews. Am J Hum Genet. 1991;49(4):855-859.

20. Poupetova H, Ledvinova J, Berna L, Dvorakova L, Kozich V, Elleder M. The birth prevalence of lysosomal storage disorders in the Czech Republic: comparison with data in different populations. $J$ Inherit Metab Dis. 2010;33(4):387-396.

21. Mehta A. Epidemiology and natural history of Gaucher's disease. Eur J Intern Med. 2006;17 Suppl:S2-S5.

22. Horowitz M, Pasmanik-Chor M, Borochowitz Z, et al. Prevalence of glucocerebrosidase mutations in the Israeli Ashkenazi Jewish population. Hum Mutat. 1998;12(4):240-244.

23. Aviner S, Garty BZ, Rachmel A, et al. Type 2 Gaucher disease occurs in Ashkenazi Jews but is surprisingly rare. Blood Cells Mol Dis. 2009;43(3):294-297.

24. Hruska KS, LaMarca ME, Scott CR, Sidransky E. Gaucher disease: mutation and polymorphism spectrum in the glucocerebrosidase gene (GBA). Hum Mutat. 2008;29(5):567-583.

25. Grabowski GA, Kolodny EH, Weinreb NJ, et al. Gaucher disease: phenotypic and genetic variation. In: Scriver C, Beudet AL, Sly WS, et al, editors. The Online Metabolic and Molecular Bases of Inherited Disease - OMMBID. Available at: www.ommbid.com. New York, McGraw-Hill, Chap 146.1. September 23, 2005. Accessed 27 Mar 2011.

26. Diaz GA, Gelb BD, Risch N, et al. Gaucher disease: the origins of the Ashkenazi Jewish N370S and 84GG acid beta-glucosidase mutations. Am J Hum Genet. 2000;66(6):1821-1832.

27. Lacerda L, Amaral O, Pinto R, Oliveira P, Aerts J, Sa Miranda MC. Gaucher disease: N370S glucocerebrosidase gene frequency in the Portuguese population. Clin Genet. 1994;45(6):298-300.

28. Dionisi-Vici C, Rizzo C, Burlina AB, et al. Inborn errors of metabolism in the Italian pediatric population: a national retrospective survey. J Pediatr. 2002;140(3):321-327.

29. Poorthuis BJ, Wevers RA, Kleijer WJ, et al. The frequency of lysosomal storage diseases in The Netherlands. Hum Genet. 1999;105(1-2): $151-156$.

30. Tsuji S, Choudary PV, Martin BM, et al. A mutation in the human glucocerebrosidase gene in neuronopathic Gaucher's disease. $N$ Engl J Med. 1987;316(10):570-575. 
31. Eto Y, Ida H. Clinical and molecular characteristics of Japanese Gaucher disease. Neurochem Res. 1999;24(2):207-211.

32. Uyama E, Takahashi K, Owada M, et al. Hydrocephalus, corneal opacities, deafness, valvular heart disease, deformed toes and leptomeningeal fibrous thickening in adult siblings: a new syndrome associated with beta-glucocerebrosidase deficiency and a mosaic population of storage cells. Acta Neurol Scand. 1992;86(4):407-420.

33. Kaplan P, Andersson HC, Kacena KA, Yee JD. The clinical and demographic characteristics of nonneuronopathic Gaucher disease in 887 children at diagnosis. Arch Pediatr Adolesc Med. 2006;160(6): 603-608.

34. Fairley C, Zimran A, Phillips M, et al. Phenotypic heterogeneity of N370S homozygotes with type I Gaucher disease: an analysis of 798 patients from the ICGG Gaucher Registry. $J$ Inherit Metab Dis. 2008;31(6):738-744.

35. Stone DL, Tayebi N, Orvisky E, Stubblefield B, Madike V, Sidransky E. Glucocerebrosidase gene mutations in patients with type 2 Gaucher disease. Hum Mutat. 2000;15(2):181-188.

36. Liou B, Grabowski GA. Participation of asparagine 370 and glutamine 235 in the catalysis by acid beta-glucosidase: the enzyme deficient in Gaucher disease. Mol Genet Metab. 2009;97(1):65-74.

37. Liou B, Kazimierczuk A, Zhang M, Scott CR, Hegde RS, Grabowski GA. Analyses of variant acid beta-glucosidases: effects of Gaucher disease mutations. J Biol Chem. 2006;281(7):4242-4253.

38. Grabowski GA, Dinur T, Osiecki KM, Kruse JR, Legler G, Gatt S. Gaucher disease types 1,2, and 3: differential mutations of the acid beta-glucosidase active site identified with conduritol B epoxide derivatives and sphingosine. Am J Hum Genet. 1985;37(3):499-510.

39. Grabowski GA, Goldblatt J, Dinur T, et al. Genetic heterogeneity in Gaucher disease: physicokinetic and immunologic studies of the residual enzyme in cultured fibroblasts from non-neuronopathic and neuronopathic patients. Am J Med Genet. 1985;21(3):529-549.

40. Christomanou H, Aignesberger A, Linke RP. Immunochemical characterization of two activator proteins stimulating enzymic sphingomyelin degradation in vitro. Absence of one of them in a human Gaucher disease variant. Biol Chem Hoppe-Seyler. 1986;367(9):879-890.

41. Christomanou H, Chabas A, Pampols T, Guardiola A. Activator protein deficient Gaucher's disease. A second patient with the newly identified lipid storage disorder. Klinische Wochenschrift. 1989;67(19): 999-1003.

42. Pampols T, Pineda M, Giros ML, et al. Neuronopathic juvenile glucosylceramidosis due to sap-C deficiency: clinical course, neuropathology and brain lipid composition in this Gaucher disease variant. Acta Neuropathol. 1999;97(1):91-97.

43. Tylki-Szymanska A, Czartoryska B, Vanier MT, et al. Non-neuronopathic Gaucher disease due to saposin C deficiency. Clin Genet. 2007; 72(6):538-542.

44. Van Meer G, Wolthoorn J, Degroote S. The fate and function of glycosphingolipid glucosylceramide. Philos Trans $R$ Soc Lond B Biol Sci. 2003;358(1433):869-873.

45. Futerman AH, Pagano RE. Determination of the intracellular sites and topology of glucosylceramide synthesis in rat liver. Biochem $J$. 1991;280(Pt 2):295-302.

46. Jeckel D, Karrenbauer A, Burger KN, van Meer G, Wieland F. Glucosylceramide is synthesized at the cytosolic surface of various Golgi subfractions. J Cell Biol. 1992;117(2):259-267.

47. Curtino JA, Caputto R. Enzymatic synthesis of glucosylsphingosine by rat brain microsomes. Lipids. 1972;7(8):525-527.

48. Yamaguchi Y, Sasagasako N, Goto I, Kobayashi T. The synthetic pathway for glucosylsphingosine in cultured fibroblasts. $J$ Biochem. 1994;116(3):704-710.

49. Conradi NG, Sourander P, Nilsson O, Svennerholm L, Erikson A. neuropathology of the Norrbottnian type of Gaucher disease. Morphological and biochemical studies. Acta Neuropathol. 1984;65(2):99-109.

50. Sidransky E. Gaucher disease: complexity in a "simple" disorder. Mol Genet Metab. 2004;83(1-2):6-15.
51. Korkotian E, Schwarz A, Pelled D, Schwarzmann G, Segal M, Futerman AH. Elevation of intracellular glucosylceramide levels results in an increase in endoplasmic reticulum density and in functional calcium stores in cultured neurons. J Biol Chem. 1999;274(31):21673-21678.

52. Pelled D, Trajkovic-Bodennec S, Lloyd-Evans E, Sidransky E, Schiffmann R, Futerman AH. Enhanced calcium release in the acute neuronopathic form of Gaucher disease. Neurobiol Dis. 2005;18(1):83-88.

53. Jmoudiak M, Futerman AH. Gaucher disease: pathological mechanisms and modern management. Br J Haematol. 2005;129(2):178-188.

54. Barak V, Acker M, Nisman B, et al. Cytokines in Gaucher's disease. Eur Cytokine Network. 1999;10(2):205-210.

55. Van Breemen MJ, de Fost M, Voerman JS, et al. Increased plasma macrophage inflammatory protein (MIP)-1alpha and MIP-1beta levels in type 1 Gaucher disease. Acta Biochim Biophys. 2007;1772(7):788-796.

56. Yoshino M, Watanabe Y, Tokunaga Y, et al. Roles of specific cytokines in bone remodeling and hematopoiesis in Gaucher disease. Pediatr Int. 2007;49(6):959-965.

57. Costello R, O'Callaghan T, Sebahoun G. Gaucher disease and multiple myeloma. Leuk Lymphoma. 2006;47(7):1365-1368.

58. Burrow TA, Cohen MB, Bokulic R, et al. Gaucher disease: progressive mesenteric and mediastinal lymphadenopathy despite enzyme therapy. J Pediatr. 2007;150(2):202-206.

59. Sobreira E, Pires RF, Cizmarik M, Grabowski GA. Phenotypic and genotypic heterogeneity in Gaucher disease type 1: a comparison between Brazil and the rest of the world. Mol Genet Metab. 2007;90(1):81-86.

60. Beutler E, Demina A, Laubscher K, et al. The clinical course of treated and untreated Gaucher disease. A study of 45 patients. Blood Cells Mol Dis. 1995;21(2):86-108.

61. Taddei TH, Kacena KA, Yang M, et al. The underrecognized progressive nature of N370S Gaucher disease and assessment of cancer risk in 403 patients. Am J Hematol. 2009;84(4):208-214.

62. Biegstraaten M, van Schaik IN, Aerts JM, Hollak CE. 'Non-neuronopathic' Gaucher disease reconsidered. Prevalence of neurological manifestations in a Dutch cohort of type I Gaucher disease patients and a systematic review of the literature. J Inherit Metab Dis. 2008;31(3): 337-349.

63. Sidransky E, Nalls MA, Aasly JO, et al. Multicenter analysis of glucocerebrosidase mutations in Parkinson's disease. $N$ Engl J Med. 2009;361(17):1651-1661

64. Nichols WC, Pankratz N, Marek DK, et al. Mutations in GBA are associated with familial Parkinson disease susceptibility and age at onset. Neurology. 2009;72(4):310-316.

65. Gan-Or Z, Giladi N, Rozovski U, et al. Genotype-phenotype correlations between GBA mutations and Parkinson disease risk and onset. Neurology. 2008;70(24):2277-2283.

66. Bultron G, Kacena K, Pearson D, et al. The risk of Parkinson's disease in type 1 Gaucher disease. J Inherit Metab Dis. 2010;33(2): 167-173.

67. Tayebi N, Walker J, Stubblefield B, et al. Gaucher disease with parkinsonian manifestations: does glucocerebrosidase deficiency contribute to a vulnerability to parkinsonism? Mol Genet Metab. 2003;79(2):104-109.

68. Weinreb NJ, Deegan P, Kacena KA, et al. Life expectancy in Gaucher disease type 1. Am J Hematol. 2008;83(12):896-900.

69. Mistry P, Germain DP. Phenotype variations in Gaucher disease. Rev Med Interne. 2006;27 Suppl 1:S3-S10.

70. Mignot C, Gelot A, Bessieres B, et al. Perinatal-lethal Gaucher disease. Am J Med Genet A. 2003;120A(3):338-344.

71. Mignot C, Doummar D, Maire I, De Villemeur TB. Type 2 Gaucher disease: 15 new cases and review of the literature. Brain Dev. 2006;28(1): $39-48$.

72. Dreborg S, Erikson A, Hagberg B. Gaucher disease-Norrbottnian type. I. General clinical description. Eur J Pediatr. 1980;133(2): 107-118.

73. Goker-Alpan O, Wiggs EA, Eblan MJ, et al. Cognitive outcome in treated patients with chronic neuronopathic Gaucher disease. J Pediatr. 2008;153(1):89-94. 
74. Goker-Alpan O, Schiffmann R, Park JK, Stubblefield BK, Tayebi N, Sidransky E. Phenotypic continuum in neuronopathic Gaucher disease: an intermediate phenotype between type 2 and type 3. J Pediatr. 2003; 143(2):273-276.

75. Grabowski GA, Kacena K, Cole JA, et al. Dose-response relationships for enzyme replacement therapy with imiglucerase/alglucerase in patients with Gaucher disease type 1. Genet Med. 2009;11(2):92-100.

76. Hughes D, Cappellini MD, Berger M, et al. Recommendations for the management of the haematological and onco-haematological aspects of Gaucher disease. Br J Haematol. 2007;138(6):676-686.

77. Martins AM, Valadares ER, Porta G, et al. Recommendations on diagnosis, treatment, and monitoring for Gaucher disease. J Pediatr. 2009;155(4 Suppl):S10-S18.

78. Pastores GM, Weinreb NJ, Aerts H, et al. Therapeutic goals in the treatment of Gaucher disease. Semin Hematol. 2004;41(4 Suppl 5):4-14.

79. Vellodi A, Tylki-Szymanska A, Davies EH, et al. Management of neuronopathic Gaucher disease: revised recommendations. J Inherit Metab Dis. 2009;32(5):660-664.

80. Weinreb NJ, Aggio MC, Andersson HC, et al. Gaucher disease type 1: revised recommendations on evaluations and monitoring for adult patients. Semin Hematol. 2004;41(4 Suppl 5):15-22.

81. Weinreb NJ, Cappellini MD, Cox TM, et al. A validated disease severity scoring system for adults with type 1 Gaucher disease. Genet Med. 2010;12(1):44-51.

82. Andersson H, Kaplan P, Kacena K, Yee J. Eight-year clinical outcomes of long-term enzyme replacement therapy for 884 children with Gaucher disease type 1. Pediatrics. 2008;122(6):1182-1190.

83. Weinreb NJ, Charrow J, Andersson HC, et al. Effectiveness of enzyme replacement therapy in 1028 patients with type 1 Gaucher disease after 2 to 5 years of treatment: a report from the Gaucher Registry. Am J Med. 2002;113(2):112-119.

84. Brumshtein B, Salinas P, Peterson B, et al. Characterization of gene-activated human acid-beta-glucosidase: crystal structure, glycan composition, and internalization into macrophages. Glycobiology. 2010; 20(1):24-32.

85. Zimran A, Altarescu G, Philips M, et al. Phase $1 / 2$ and extension study of velaglucerase alfa replacement therapy in adults with type 1 Gaucher disease: 48-month experience. Blood. 2010;115(23):4651-4656.

86. Zimran A, Loveday K, Fratazzi C, Elstein D. A pharmacokinetic analysis of a novel enzyme replacement therapy with gene-activated human glucocerebrosidase (GA-GCB) in patients with type 1 Gaucher disease. Blood Cells Mol Dis. 2007;39(1):115-118.

87. Shaaltiel Y, Bartfeld D, Hashmueli S, et al. Production of glucocerebrosidase with terminal mannose glycans for enzyme replacement therapy of Gaucher's disease using a plant cell system. Plant Biotechnol J. 2007;5(5):579-590.

88. Ruiz J, Barzegar S, Clarke A, et al. Antigenic differences in patients receiving velaglucerase alfa or imiglucerase. Presented at the 6th Annual Lysosomal Disease Network World Symposium, Miami, FL, February 10-12, 2010.
89. Radin NS. Preparative isolation of cerebrosides (galactosyl and glucosyl ceramide). J Lipid Res. 1976;17(3):290-293.

90. Cox T, Lachmann R, Hollak C, et al. Novel oral treatment of Gaucher's disease with N-butyldeoxynojirimycin (OGT 918) to decrease substrate biosynthesis. Lancet. 2000;355(9214):1481-1485.

91. Elstein D, Hollak C, Aerts JM, et al. Sustained therapeutic effects of oral miglustat (Zavesca, N-butyldeoxynojirimycin, OGT 918) in type I Gaucher disease. J Inherit Metab Dis. 2004;27(6):757-766.

92. Elstein D, Dweck A, Attias D, et al. Oral maintenance clinical trial with miglustat for type I Gaucher disease: switch from or combination with intravenous enzyme replacement. Blood. 2007;110(7): 2296-2301.

93. Pastores GM, Elstein D, Hrebicek M, Zimran A. Effect of miglustat on bone disease in adults with type 1 Gaucher disease: a pooled analysis of three multinational, open-label studies. Clin Ther. 2007;29(8): 1645-1654

94. Patterson MC, Vecchio D, Prady H, Abel L, Wraith JE. Miglustat for treatment of Niemann-Pick $\mathrm{C}$ disease: a randomised controlled study. Lancet Neurol. 2007;6(9):765-772.

95. Schiffmann R, Fitzgibbon EJ, Harris C, et al. Randomized, controlled trial of miglustat in Gaucher's disease type 3. Ann Neurol. 2008;64(5): 514-522.

96. Capablo JL, Franco R, de Cabezon AS, Alfonso P, Pocovi M, Giraldo P. Neurologic improvement in a type 3 Gaucher disease patient treated with imiglucerase/miglustat combination. Epilepsia. 2007;48(7):1406-1408.

97. Champion H, Ramaswami U, Imrie J, et al. Dietary modifications in patients receiving miglustat. J Inherit Metab Dis. 2010 Sep 16. [Epub ahead of print].

98. Hollak CE, Hughes D, van Schaik IN, Schwierin B, Bembi B Miglustat (Zavesca) in type 1 Gaucher disease: 5-year results of a post-authorisation safety surveillance programme. Pharmacoepidemiol Drug Saf. 2009;18(9):770-777.

99. Lukina E, Watman N, Arreguin EA, et al. A phase 2 study of eliglustat tartrate (Genz-112638), an oral substrate reduction therapy for Gaucher disease type 1. Blood. 2010;116(6):893-899.

100. Lukina E, Watman N, Avila Arreguin E, et al. Improvement in hematological, visceral, and skeletal manifestations of Gaucher disease type 1 with oral eliglustat tartrate (Genz-112638) treatment: two-year results of a Phase 2 study. Blood. 2010;116(20):4095-4098.

101. Peterschmitt MJ, Burke A, Blankstein L, et al. Safety, tolerability, and pharmacokinetics of eliglustat tartrate (Genz-112638) after single doses, multiple doses, and food in healthy volunteers. J Clin Pharmacol. 2010 Sep 23. [Epub ahead of print].

102. McEachern KA, Fung J, Komarnitsky S, et al. A specific and potent inhibitor of glucosylceramide synthase for substrate inhibition therapy of Gaucher disease. Mol Genet Metab. 2007;91(3):259-267.
Pediatric Health, Medicine and Therapeutics

\section{Publish your work in this journal}

Pediatric Health, Medicine and Therapeutics is an international, peerreviewed, open access journal publishing original research, reports, editorials, reviews and commentaries. All aspects of health maintenance preventative measures and disease treatment interventions are addressed within the journal. Practitioners from all disciplines are invited to submit

\section{Dovepress}

their work as well as healthcare researchers and patient support groups. The manuscript management system is completely online and includes a very quick and fair peer-review system. Visit http://www.dovepress. $\mathrm{com} /$ testimonials.php to read real quotes from published authors. 